\title{
11 Surface Treatments
}

Surface treatments are usually carried out at the end of the drying phase, once the pottery vessels reach the critical point and are in what is known as the leather-hard stage. At this time, the paste is relatively dry and the pottery is consistent enough to undertake further actions. However, it is also wet enough to be workable and sensitive to the technical gestures made by the potters. The most common surface treatments applied to prehistoric vessels consist in the application of slips and polishing. These actions have also effects on the behaviour of the paste during the firing process as well as in the properties of the end product.

Other actions such as decorations may be included in this phase of the chaine opératoire since both surface treatments and decorations are applied once the pottery is dried. Anyway, it should be stated that the colour of pottery, although clearly decorative and perceptible, does not have to be included in the surface treatment phase. In this way, some traditional societies establish a clear division between the decoration and the colour of the vessels. The latter results mainly from the firing process and is therefore not considered as part of the decoration (Krause, 1984).

Another action that alters the surface of the pottery and is related to decoration is the application of paintings on the surface of the vessels. Pigments are usually selected from organic substances or iron oxide nodules (e.g., hematite or magnetite) and copper oxide which occur naturally in the environment. As with some slips, these minerals have to be crushed and diluted in water or fats, among other components, before its application. Thus, the use of pigments such as hematite provides a characteristic red colour to the pottery surface (Capel et al., 2006; Krause, 1984). Beyond their high visual impact these pigments do not involve significant changes, neither in the physical properties of the paste nor the final product obtained.

The most common surface treatment involving mud raw materials is the slip. Slips consist in clay particles in aqueous solution that are usually applied on the ceramic surface once the paste has reached the leather-hard stage and before firing. Thus, this action, when carried out on the unfired ceramic body, rehydrates the outermost layers of the pottery. This rehydration can lead to cracks during the subsequent drying or firing processes as a consequence of the mechanical discontinuity between the paste and the slip. The vessel is better dried and fired through the gradual application of several thin layers of slip preferably performed with the clay formerly used to shape the pottery. The use of macro-porous pastes (e.g., calcareous) favours the application of the slip by its infiltration through the pores. A higher infiltration facilitates the adherence of slips due to different dilatometric behaviour of the materials. Furthermore, slip may be an ideal media on which to apply other surface treatments such as polishing. Thus, the final appearance of the pottery mainly depends on the type of raw materials used to produce the slip (e.g., iron and calcite content), the way slips are prepared and the techniques used in their application. Other variables involved are, for example, the subsequent firing atmosphere, the thickness of the 
vessel walls and the colour of the pottery (Banning, 2005; Capel et al., 2006; Cuomo Di Caprio, 1985; Echallier, 1984; Gibson and Woods, 1990; Gómez and Doherty, 2000; Matson, 1989; Morales, 2005; Reid, 2001; Turbanti, 2004).

Slips may have a variable thickness typically ranging between 30 and $80 \mu \mathrm{m}$, although sometimes they measure less than $10 \mu \mathrm{m}$. On the one hand, the mere immersion of the vessel in liquefied suspension of clay particles in water can be considered as a slip. Nevertheless, this kind of application is very difficult to identify in the archaeological record due to the small amount of clay that is incorporated and the degradation of the surface of the vessels during its burial (Echallier, 1984). On the other hand, there are other slips that are easier to detect that consist on the application of a thicker layer of more or less purified clay. In addition, these slips may contain high amounts of iron ore (e.g., $\mathrm{Fe}_{2} \mathrm{O}_{3}, \mathrm{Fe}_{3} \mathrm{O}_{4}$ ), as occurs in the case of ceramics from the Serraferlicchio and Pantalica cultures during the Copper and Bronze Age in Sicily that have up to $15 \%$ in iron content (Cuomo Di Caprio, 1985). Moreover, Neolithic ceramics from southern Iberia can even reach up to $35 \%$ of $\mathrm{Fe}_{2} \mathrm{O}_{3}$ (Capel et al., 2006).

The use of iron-rich slip (Fig. 11.1) does not entail disadvantages resulting from a differential thermal expansion with the ceramic body, since their thermal behaviour is relatively analogous. The crushed iron ore added to clay and water provides a reddish or red-orange chromatic scheme to the surfaces of the pottery that may be also brown or dark-brown if coal is also added. The brightness and reddish colour of the slips can be enhanced through the addition of higher amounts of iron oxide to the mixture. In this way, the introduction of iron-rich minerals and, to a lesser extent, titanium oxides or calcareous materials causes physical changes that alter the appearance of the ceramics (Capel et al., 2006; Cuomo Di Caprio, 1985; Morales, 2005; Schiffer and Skibo, 1987).

While the preparation of the slip does not entail much difficulty, the drying phase may represent a delicate operation. As noted, the application of slip involves an extra drying phase in the pottery, thus increasing the time needed to develop the production. The clay added to the mixture, usually in higher proportion than the iron ore, provides enough adhesion to firmly adhere the slip to the surface of the pottery. Thus, the slip does not come off in later stages of the production process or during the use of the vessels. When the clay used in the manufacture of the pottery is fine enough or intensely purified, the slips can be also carried out using the former raw material. However, differences in the grain-size and the composition of both, paste and slip, will provide a different coloration between the body and the surface of the vessel. In cases in which coarse clays are used to shape the pottery other more suitable raw materials can be selected to perform this surface treatment. In this case, the use of clays with different composition results also in a diverse coloration (Cuomo Di Caprio, 1985).

Optical methods such as binocular and the petrographic microscopes can be successfully used to approach the surface treatments applied in the manufacture of ceramics. In some cases, slips consisting in several layers of very fine clay applied 
one over another can be even observed. This technological choice promotes a better adhesion of the slip to the surface of the pottery, preventing its removal during the subsequent drying and firing processes. In any case, slips can be further approached and characterised with accuracy in chemical, mineralogical and textural terms through archaeometric methods such as petrography, image analysis, SEM-EDX and XRD (Buxeda et al., 1995; Capel et al., 2006; Gibson and Woods, 1990; Gliozzo et al., 2008; Gómez and Doherty, 2000; Tite, 2008; Tite et al., 1982; Peterson, 2009; Tschegg, 2009; Velde, 2005).
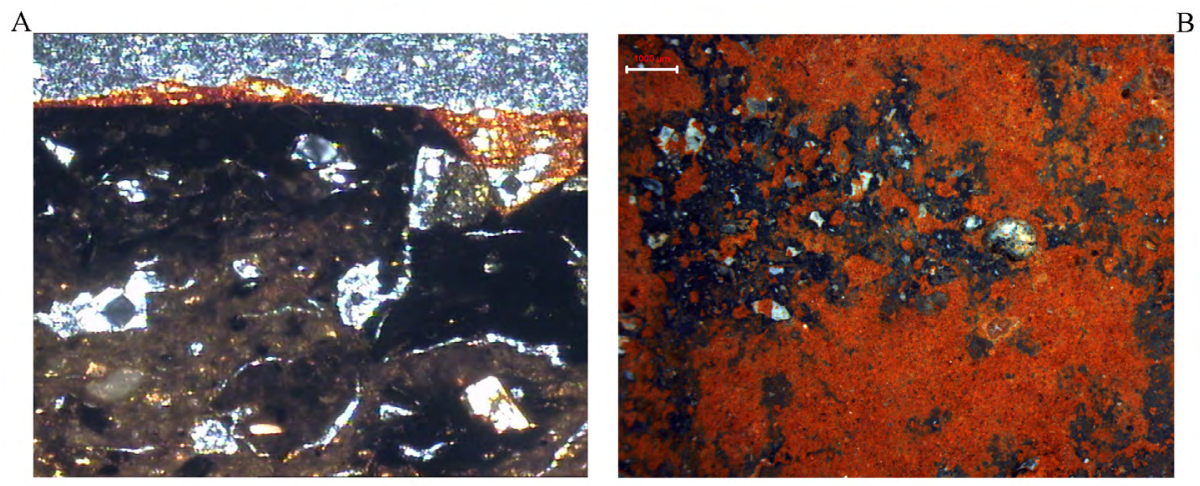

Figure 11.1: Iron-rich slip detected in a pottery. A) Thin section photomicrograph taken in cross polarised light showing a fine-textured and thin reddish slip filling the pores in the vessel surface (Image width $=2.3 \mathrm{~mm}$ ). B) Photomicrograph taken with binocular microscope of the same red slip observed on the surface of the vessel (Image width $=11.2 \mathrm{~mm}$ ).

As seen, slips are mainly formed by raw materials very rich in clay minerals and, hence, in alumina. Although the presence of inclusions in the slip is usually very low, aplastic components are not always totally absent. Sometimes there are vessels with thicker slips (> $300 \mu \mathrm{m}$ thick) which may also have coarser textures (Fig. 11.2). The texture and porosity of the slip are useful parameters to distinguish this surface treatment from the ceramic body. These features permit to identify the slips and avoid confusions with postdepositional processes that also alter the surface of the vessel (Velde, 2005; Tschegg, 2009).

Slips usually have a low amount of inclusions predominantly sorted below the medium sand fraction $(<500 \mu \mathrm{m})$. These textural features can contrast significantly with the frequency and size of the inclusions occurring in the paste, where the size of most particles is usually above this range. Thus, some studies (e.g., Capel et al., 1982) have identified the presence of slips on the basis of textural criteria. Thus, the occurrence in the surface of the pottery of a distinctive fine-textured layer of clay with a very low frequency of inclusions may be an evidence of this surface treatment. In 
these cases the raw materials were not intensely purified but are better prepared than the paste used to shape the vessels.

These coarser slips are distinguishable with petrographic microscope and, on many occasions, with binocular microscope (Cuomo Di Caprio, 1985). However, there are some constraints to differentiate through textural criteria the presence of slips when ceramic bodies are also fine-textured. Due to this limitation, the identification of slips by means of textural parameters is restricted to cases in which the ceramic body has a medium or coarse texture ( $>500 \mu \mathrm{m}$, coarse sand to granules). Also, the identification of slips through binocular microscope on the basis of different colour gradients in the vessel section may lead to confusion. Often colour variations occurring in the outer margins and surfaces of the vessels seem to be related to slips. However, these chromatic variations may be also associated with sudden changes in the atmosphere during the firing process. In any case, both the existence of chromatic variations along the section together with the textural features of the matrix and the surface layers can be considered in an attempt to identify the presence of slips in the vessels.
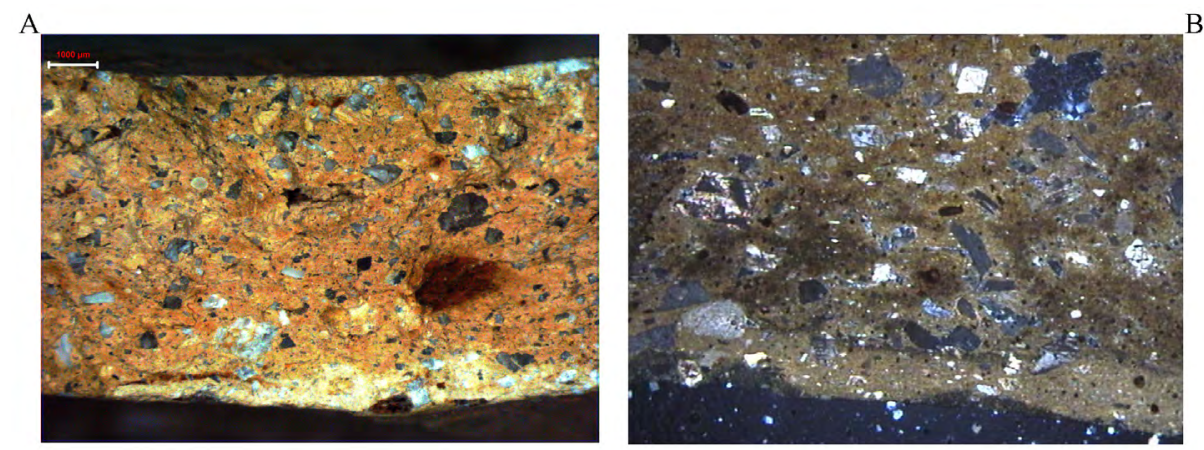

Figure 11.2: Thick coarse-textured slip. A) Photomicrograph taken with binocular microscope showing the chromatic and textural differences between the slip and the ceramic body (Image width $=10.2 \mathrm{~mm}$ ). B) Thin section photomicrograph in cross polarised light (Image width $=4.6 \mathrm{~mm}$ ) of the same pottery vessel in which the textural divergences are evident.

In addition to slips, it is possible to identify by optical means other finishing surface treatments in vessels such as polishing (Fig. 11.3 A). Burnishing the surface of the pottery provokes a perfect parallel alignment along the vessel margins of those inclusions which are closest to the surface (Fig. 11.3 B). This arrangement of the particles evidences that the polished was performed when the pottery was in leatherhard stage. Polishing the surface of the pottery using a hard smooth tool promotes the orientation of the lamellar structure of the clay minerals, thus providing glossy surfaces that are quite similar to metallic lustres. 

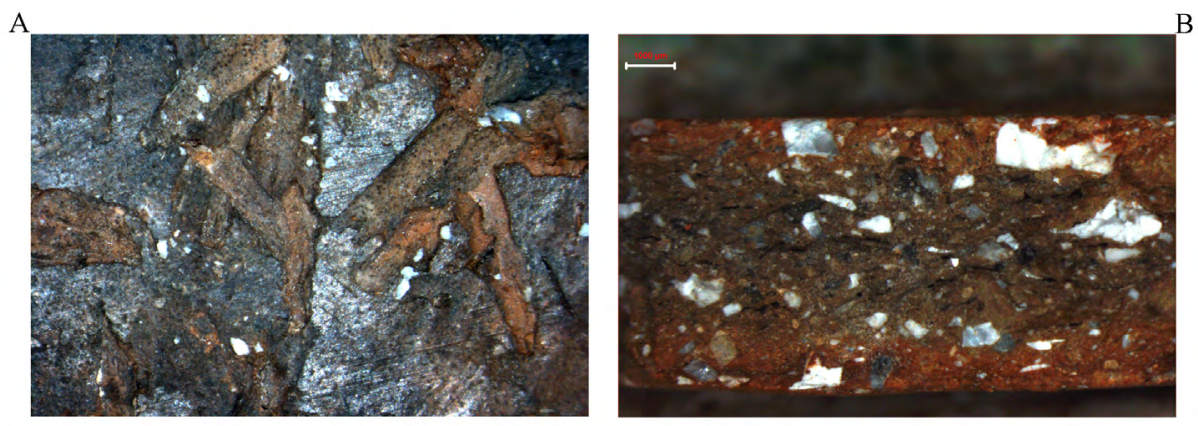

Figure 11.3: Photomicrographs taken with binocular microscope showing the effect of burnish the surface of the ceramic. A) Glossy metallic lustre. B) Parallel alignment of the temper along the vessel margins due to surface treatments such as paddle and burnish (Image width $=11 \mathrm{~mm}$ ). 